INTERNATIONAL JOURNAL OF

SYSTEMATIC BACTERIOLOGY

Vol. 19, No. 2 April 1969

pp. $119-122$

Copyright 1969, Iowa State University Press

\title{
A-PROPOS DE LA CLASSIFICATION DES CORYNEBACTERIA ANAÉROBIES
}

\author{
A. R. Prévot \\ Institut Pasteur, Paris
}

Dans un article récent paru dans ce journal, Zierdt, Webster et Rude (1) proposent la réduction du nombre des especes anaérobies du genre Corynebacterium, ce qui met en cause les essais de classification de ces bactéries que j'ai proposés en 1938 d'abord, puis en 1961, 1966 et 1967 (2).

Les essais que j'ai proposés sont impliqués déjà dans les différences phénotypiques observées par les auteurs qui ont décrit les onze especes anaérobies de ce genre. Nous avons retrouvés au cours de vingt-cinq années d'étude de plus de 400 souches de Corynebacterium anaérobie les mêmes différences phénotypiques et en avons décrit de nouvelles. Mais jusqu'en 1963 nous avons prudemment considéré les onze espèces décrites comme phénotypiques, donc susceptibles de réduction après que la connaissance de leur génome ait été réalisée. Or en 1963 (3) j'ai conseillé a Bouisset, Breuillaud et Michel d'entreprendre des recherches sur le génome de ces especes par le truchement du quotient de Shapiro-Chargaff de leur ADN. Les résultats de cette étude permettent à la fois de confirmer l'hétérogénéité génotypique de ces especes et aussi d'esquisser un nouveau regroupement que les caracteres phénotypiques étaient incapable de faire prévoir.

Cette hétérogénéité et cette possibilité de regroupement vont a l'encontre des opinions de Zierdt, Webster et Rude puisque celle-ci est étayée uniquement sur des caracteres phénotypiques et sérologiques.

Voici briévement résumés, les résultats de ces auteurs, que nous avons intégrés dans le chapitre concernant les Corynebacterium anaérobies de notre dernier traité (2) (non cité par ces auteurs).

Le quotient $\frac{A+T}{G+C}$ de 60 souches de Corynebacterium a permis le regroupement suivant: 
$1^{\circ}$ - Quotient élevé: Corynebacterium pyogenes $=1,07 \mathrm{chef}$

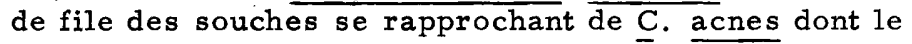
quotient est 1,08 .

$2^{\circ}$ - Quotient moyen: C. avidum $=0,93$

C. granulosum $=0,93$

$\bar{C}$. liquefaciens $=0,94$

$\overline{\text { C. }} \overline{\text { hepatodystrophicans }}=0,91$

constituant peut-être un génotype autonome nettement différent de $\underline{\text { C. acnes. }}$

$3^{\circ}$ - Quotient faible: C. diphtheroides $=0,88$

C. $\overline{\text { lymphophilum }}=0,85$

C. renale $=0,89$

ce groupe est intermédiaire entre le groupe de $C$. avidum et le groupe suivant.

$4^{\circ}$ - Quotient très faible: C. parvum $=0,72$.

A ce groupe il sera peut être possible de rattacher l'importante espece C. anaerobium, dont l'étude génotypique $n^{\prime}$ est pas terminée mais semble se rapprocher de celle de $\underline{C}$. parvum.

Discussion et conclusions: La connaissance de l'espèce bactérienne a fait un progrès considérable depuis que Belozerskig, Shapiro, Chargaff, Lee et Barbu, Sébald, Gasser, ainsi que d'autres auteurs ont élaboré les techniques permettant $1^{\prime}$ étude quantitative sur la constitution de l'ADN des bactéries. Tout progrès dans la classification des especes ne pourra être réalisé que s'il est conforme aux données nouvelles concernant leur génome. Dans le cas des Corynebacterium anaérobies, seule l'espece proposée $\underline{C}$. pyogenes se rapproche du génotype de $\mathrm{C}$. acnes. Les autres s'en éloignent manifestement et ceci permet d'en distinguer 3 groupes:

$1^{\circ}$ - Le groupe $\underline{\text { C. avidum, }} \underline{\text { C. granulosum, }} \underline{\text { C. liquefaciens, }}$ C. hepatodystrophicans.

$2^{\circ}$ - Le groupe $\underline{\text { C. diphtheroides, }}$ C. lymphophilum, C. renale. $3^{\circ}$ - Le groupe $\underline{\text { C. parvum, }}$ C. anaerobium.

En dehors de leur intérêt purement théorique, ces considérations prennent une importance considérable en pathol- 
ogie et en thérapeutique, car le groupe C. acnes est peu ou pas pathogène et ne nécessite pas une thẻrapeutique spécifique alors qu'au contraire, dans les 3 autres groupes on rencontre des especes très pathogenes pour l'homme et les animaux, qu'on doit traiter par une antibiothérapie très spécifique, les corynébactérioses anaérobies non correctement traitées pouvant provoquer une haute mortalité (comme par exemple la maladie de Whipple (4), mortelle au taux de $100 \%$, actuellement guérie au taux de $100 \%$, grace a 1' antibiotique révélé par l'antibiogramme anaérobie pratiqué avec la souche isolée des ganglions mésentériques ou de la lamina propria.

\section{REFERENCES}

1. Zierdt, C.H., C. Webster, et W.S. Rude. 1968. Int. J. Syst. Bact. 18:33.

2. Prevot, A. R., A. Turpin et P. Kaiser. 1967. Les Bactéries anaérobies. 1 vol. 2280 pp., Dunod Editeur Paris.

3. Bouisset, L., J. Breuillaud et G. Michel. 1963. Ann. Inst. Pasteur, 1963:104.

4. Prevot, A. R. 1965. Monde médical. 75:961. 
